\title{
Effect of Deformation of Red Cell on Nutritional Transport in Capillary-Tissue Exchange System
}

\author{
Rekha Bali $^{1}$, Swati Mishra ${ }^{1}$, Mamta Mishra ${ }^{2 *}$ \\ ${ }^{1}$ Department of Mathematics, Harcourt ButlerTechnological Institute, Kanpur, India \\ ${ }^{2}$ Department of Mathematics, School of Applied Sciences, NSIT, New Delhi, India \\ E-mail:dr.rekhabali@rediffmail.com, swatimishra1982@yahoo.co.in \\ Received October 27, 2010; revised August 9, 2011; accepted August 16, 2011
}

\begin{abstract}
A mathematical analysis of a model for nutritional exchange in a capillary-tissue exchange system is presented in this paper. This model consists of a single file flow of red blood cell in capillary when diameter of red blood cell is greater than tube diameter. In this case, the cell must be deformed. Due to concentration gradients, the dissolved nutrient in substrate diffuses into surrounding tissue. Introducing approximations of the lubrication theory, squeezing flow of plasma in between the gap between cell and capillary wall have been solved with the help of approximate mathematical techniques. The computational results for concentration of dissolved nutrients, diffusive flux and normal component of velocity have been presented and discussed through graphs. We have also shown the effect of deformation parameter and permeability on these results.
\end{abstract}

Keywords: Capillary-Tissue-Exchange, Red Blood Cell, Nutrients, Diffusion, Diffusive Flux

\section{Introduction}

A major difficulty in studying the microcirculation is the small dimensions of the blood vessels. Experimental data on Pressure, velocity, flow, shear stress, mass transfer, etc. are difficult to obtain in vivo. Hence, a model in vitro experiment that obeys geometric and dynamic similarity has been very useful. Model experiments, however, are sometimes impractical, tedious or too difficult to carry out. As such, mathematical modeling is an attractive alternative. The most physiologically important function of the circulation of blood through capillaries is to supply nutrients to every living cell of the organism and also to remove various waste products from every cell. This function is fulfilled by the transport of the various components of blood across a capillary wall into the surrounding tissue. Nutrients, dissolved in plasma, enter the tissue from capillary wall. The material is transported by convection and diffusion in the capillary, whereas in the tissue the material is transported through diffusion only as the convection velocity in the tissue is very small.

As blood flows through the capillary, the dissolved materials exchange between plasma and surrounding tissue. These materials (glucose, albumin and lactoge) present in dissolved state. Renkin [1] has considered that the solute, in plasma enters at a constant rate into the capillary from the arterial end, escapes by diffusion as the plasma in the gap between cell and wall. He has calculated the reduced concentration of the solute at the venous end and suggested that the transport of the substances across the wall is affected by the plasma flow at the interface of capillary and tissue as well as the capillary surface area, permeability and partition coefficient.

Some authors [2-4] proposed various representative models for blood in narrow capillaries. Two, three region flow models $[5,6]$ have also been developed. Tandon et al. [7] have developed a model consisting of the viscous fluid representation which is identically same as the suspending medium of the blood. Other models have also been proposed to discuss the nutrition transport in capillaries, but no work has been undertaken except Tandon et al. [8] in very narrow capillary i.e. when capillary is of radius less than diameter of red cell including the study of mass transfer which also constitute a very important aspect to analyze nutritional transport from plasma to the capillary wall [9]. Only Flow problems in very narrow capillary have been discussed till now by some authors [10-15]. While the blood flow in microvessels constitute attractive problems for mathematical analysis. As capillary size decreases, most of the cells are seemed to move 
like piston and some time quite a few of them move together, like stacked coins. In smaller capillaries, the cells enter edge wise and deform into the bullet shape with a convex leading edge. As the capillary size further decreases the sheared core flow changes to the axial train configuration. The red blood cells then move in a single file surrounded by thin plasmatic layer near the capillary wall when blood flows through the capillary whose diameter is less than that of a red cell, For such cases Lighthill assumed undeformed cell shape near the wall to be parabolic and cell deformation is depend on local pressure. Barnard et al. has assumed the cell as flexible circular sheet deformed into hollow thimble shape with isotropic tension acting on the membrane. Zarda et al. [12] considered red blood cell with axisymmetric shape for analyzing the flows in capillary at low velocity. Lin et al. [13] has done same work as Bernard but the cell was represented as a solid bullet like shape with isotropic tension acting in the cell membrane. A theoretical model is used to investigate the effect of flow velocity on motion and axisymmetric deformation of red blood cell in a capillary with an endothelial surface layer [15,16] A theoretical model is also proposed by Secomb et al. [17] to analyze the effects of the glycocalyx layer on hematocrit and resistance to blood flow in capillaries. They have considered the glycocalyx as a porous layer that resist penetration by red blood cells.

In all the above mentioned work, the problem of nutritional transport in very narrow capillaries has not been discussed. Therefore, in this paper our aim is to study the nutritional transport through the plasma, in between the cell and capillary wall, into the tissue. Lubrication theory is used to describe the squeezing flow of plasma in between the cell and tissue wall. A detailed analysis of nutritional transport has been discussed in this paper. The overall system has been modeled as two region flow and diffusion models: squeezing flow of purely viscous fluid in between cell and capillary wall and Darcy's flow of filtered plasma at the capillary tissue barrier into the porous tissue.

\section{Formulation of the Problem}

We have interoduced two dimensional cartesian geometry (Figure 1). The red cell is modeled as axisymmetric containing an incompressible fluid. Single file flow of red blood cell is considered and cell to cell interactions are neglected. The fluid film thickness $h^{\prime}$ of the plasma between the cell and the tissue wall is represented by

$$
h^{\prime}=(\alpha+\beta)^{\prime}\left(\mathrm{P}^{\prime}-\mathrm{P}_{0}^{\prime}\right)+\frac{x^{\prime 2}}{4 a}
$$

where $P^{\prime}$ is the pressure in fluid film region, $a$ is the focal length of the initially assumed shape of parabola

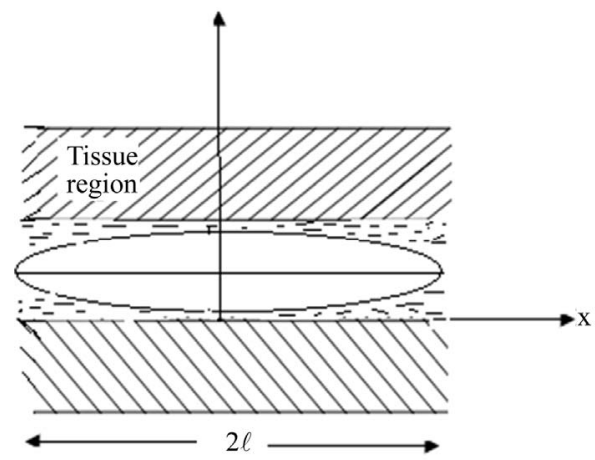

Figure 1. Diagram of single red blood cell in capillary surrounded by tissue.

and $(\alpha+\beta)^{\prime}\left(\mathrm{P}^{\prime}-\mathrm{P}_{0}^{\prime}\right)$ represent the further deformation due to increased pressure in wedge formed in between the parabola and the capillary, $U_{0}$ is the velocity of cell, $V_{0}$ is the velocity of the fluid. $\alpha$ and $\beta$ are parameters representing coefficients of small deformation in cell and wall, $\mathrm{P}_{0}^{\prime}$ is the reference pressure.

Flow region is divided into two Sub-regions:

1) Fluid Film region;

2) Porous tissue region.

\subsection{Governing Equations}

\subsubsection{Fluid Film Region}

Using the following assumptions

1) Thickness of the fluid layer between the cell and the wall is assumed to be sufficiently small so that stoke's equation can be reduced to the Reynold's equation using lubrication theory;

2) The suspending fluid is assumed to be in compressible and Newtonian.

The governing equation of motion for the flow of plasma in between the cell and capillary tissue wall are given by

$$
\begin{gathered}
\frac{\partial \mathrm{P}^{\prime}}{\partial x^{\prime}}=\mu \frac{\partial^{2} u^{\prime}}{\partial y^{\prime 2}} \\
\frac{\partial \mathrm{P}^{\prime}}{\partial y^{\prime}}=0
\end{gathered}
$$

but due to small leakage in to the porous wall we retain the continuity equation as:

$$
\frac{\partial u^{\prime}}{\partial x^{\prime}}+\frac{\partial v^{\prime}}{\partial y^{\prime}}=0
$$

where $u^{\prime}$ and $v^{\prime}$ are the velocity component along axial and transverse directions and $\mu$ is the viscosity of plasma in the capillary.

\subsubsection{Porous Tissue Region}

Following Darcy's law the axial and normal components 
of velocity in tissue region are given by

$$
\bar{u}^{\prime}=-\frac{K}{\rho} \frac{\partial \overline{\mathrm{P}}^{\prime}}{\partial x^{\prime}}, \vec{v}=-\frac{K}{\rho} \frac{\partial \overline{\mathrm{P}}^{\prime}}{\partial y^{\prime}}
$$

where $\bar{u}^{\prime}$ and $\vec{v}^{\prime}$ are the axial and transverse velocities of the fluid in the porous matrix, $K$ is the permeability of tissue.

Using these velocities in the equation of continuity, we get the Laplace's Equation for the pressure distribution in the porous tissue region as given below:

$$
\frac{\partial^{2} \overline{\mathrm{P}}^{\prime}}{\partial x^{\prime 2}}+\frac{\partial^{2} \overline{\mathrm{P}}^{\prime}}{\partial y^{\prime 2}}=0
$$

\subsection{Nutritional Transport}

\subsubsection{Fluid Film Region}

The concentration of the dissolved nutrients has been assumed to be uniform due to the mixing action of vortex ring type plasma flow in between the two cells and for very small wedge in between cell and the wall.

\subsubsection{Porous Tissue Region}

Under the admissible assumptions, the approximate diffusion equation in porous matrix is given by:

$$
v \frac{\mathrm{d} c^{\prime}}{\mathrm{d} y^{\prime}}=D^{\prime} \frac{\mathrm{d}^{2} c^{\prime}}{\mathrm{d} y^{\prime 2}}+m^{\prime}
$$

where $c^{\prime}$ the concentration of dissolved nutrients in the tissue region, $D^{\prime}$ is the diffusion coefficient and $m^{\prime}$ is the rate of production or consumption of the nutrient with in the tissue.

\subsection{Boundary and Matching Conditions}

$$
\begin{aligned}
& u^{\prime}=U_{0} \quad \text { at } \quad y^{\prime}=h^{\prime} \\
& u^{\prime}=-\sigma^{\prime} \frac{\partial u^{\prime}}{\partial y^{\prime}} \quad \text { at } \quad y^{\prime}=0 \\
& \frac{\partial \overline{\mathrm{P}}^{\prime}}{\partial x^{\prime}}=0 \quad \text { at } \quad x^{\prime}=0 \\
& \frac{\partial \overline{\mathrm{P}}^{\prime}}{\partial x^{\prime}}=0 \quad \text { at } \quad x^{\prime}=0 \\
& \overline{\mathrm{P}}^{\prime}=0 \quad \text { at } \quad x^{\prime}= \pm \ell^{\prime} \\
& \mathrm{P}^{\prime}=\mathrm{P}_{0}^{\prime} \quad \text { at } x^{\prime}= \pm \ell^{\prime} \\
& \mathrm{P}^{\prime}=\overline{\mathrm{P}}^{\prime}(x, 0) \\
& c^{\prime}=\eta c_{0} \quad \text { at } \quad y^{\prime}=0 \\
& \frac{\mathrm{d} c^{\prime}}{\mathrm{d} y^{\prime}}=0 \quad \text { at } \quad y^{\prime}=-H
\end{aligned}
$$

\subsection{Non-Dimensional Scheme}

$$
\begin{gathered}
x=\frac{x^{\prime}}{\ell} ; y=\frac{y^{\prime}}{H} ; \mathrm{P}=\frac{\mathrm{P}^{\prime}}{\rho V_{0}^{2}} ; u=\frac{u^{\prime}}{V_{0}} ; \\
\mathrm{P}_{0}=\frac{\mathrm{P}_{0}^{\prime}}{\rho V_{0}^{2}} ; v=\frac{v^{\prime}}{V_{0}} ; \mathrm{Re}=\frac{\rho V_{0} H}{\mu} ; \\
\mathrm{P} e=\frac{U_{0} H}{D^{\prime}} ; M=\frac{m^{\prime} H}{V_{0} c_{0}}
\end{gathered}
$$

where $H$ is the thickness of porous matrix, $c_{0}$ is the uniform concentration of the nutrient in the capillary, $\eta$ is the Partition coefficient.

\subsection{Governing Equation in Dimensionless Form}

Using the non-dimensional scheme, the Equations (2) to (7) are transformed as given below:

$$
\begin{aligned}
& h=\alpha^{\prime}\left(\frac{\mathrm{P}}{\mathrm{P}_{0}}-1\right)+\varepsilon x^{2} \\
& \operatorname{Re} \frac{\partial \mathrm{P}}{\partial x}=\frac{\partial^{2} u}{\partial x^{2}} \\
& \frac{H}{\ell} \frac{\partial u}{\partial x}+\frac{\partial v}{\partial y}=0 \\
& \bar{u}=-\frac{K \operatorname{Re}}{H \ell} \frac{\partial \overline{\mathrm{P}}}{\partial x} ; \bar{v}=-\frac{K \operatorname{Re}}{H \ell} \frac{\partial \overline{\mathrm{P}}}{\partial y} ; \\
& \frac{H^{2}}{\ell^{2}} \frac{\partial^{2} \bar{u}}{\partial x^{2}}+\frac{\partial^{2} \bar{v}}{\partial y^{2}}=0 \\
& v \frac{\mathrm{d} c}{\mathrm{~d} y}=\frac{\mathrm{d}^{2} c}{\mathrm{~d} y^{2}}+M .
\end{aligned}
$$

\subsection{Boundary and Matching Conditions in Dimensionless Form}

$$
\begin{array}{llr}
u=\frac{U_{0}}{V_{0}} & \text { at } & y=h \\
u=-\sigma \frac{\partial u}{\partial y} & \text { at } & y=0 \\
v=0 & \text { at } & y=h \\
\frac{\partial \mathrm{P}}{\partial x}=\frac{\partial \overline{\mathrm{P}}}{\partial x}=0 & \text { at } & x=0 \\
\mathrm{P}=\mathrm{P}_{0} & \text { at } & x= \pm \ell \\
\overline{\mathrm{P}}=0 & \text { at } & x= \pm \ell \\
\mathrm{P}(x)=\overline{\mathrm{P}}(x, 0) & \text { at } & y=0
\end{array}
$$


where

$$
\begin{array}{lll}
c=\eta & \text { at } & y=0 \\
\frac{\mathrm{d} c}{\mathrm{~d} y}=0 & \text { at } & y=-1
\end{array}
$$

$$
\begin{gathered}
\sigma H=\sigma^{\prime} \\
\alpha^{\prime}=\frac{(\alpha+\beta)^{\prime} \rho V_{0}^{2} \mathrm{P}_{0}}{H} \\
\varepsilon=\frac{\ell^{2}}{4 a H} .
\end{gathered}
$$

\subsection{Solution of the Problem}

\subsubsection{Velocity Distribution in Capillary Region}

We have solved the equation of motion using the boundary conditions $u=\frac{U_{0}}{V_{0}}$ at $y=h$ and $u=-\sigma \frac{\partial u}{\partial y}$ at $y=0$. we get the solution for axial velocity as given below:

$$
u=\operatorname{Re} \frac{\partial \mathrm{P}}{\partial x}\left[y^{2}-\frac{h^{2}(y-\sigma)}{(h-\sigma)}\right]+\frac{U_{0}(y-\sigma)}{V_{0}(h-\sigma)} .
$$

\subsubsection{Pressure Distribution in Capillary Region}

Considering velocity distribution in the equation of continuity, the pressure distribution in capillary region is obtained as

$$
\mathrm{P}=P_{0}-\frac{3 K}{H^{2} \sigma}\left(F_{5}(x)+F_{6}(x)\right)
$$

where

$$
\begin{aligned}
F_{5}(x)= & \left(F_{1}+F_{3}\right) \\
& \cdot \sum_{n=0}^{\infty} 2 E n \sinh \left\{\alpha_{n}(H+y)\right\}\left(\frac{\cos \left(\alpha_{n} x\right)+\cos \left(\alpha_{n}\right)}{\alpha_{n}^{2}}\right) \\
F_{6}(x)= & F_{2} \sum_{\mathrm{n}=0}^{\infty} 2 E n \sinh \left\{\alpha_{n}(H+y)\right\}\left(\frac{x^{4}-1}{12}-\frac{\alpha_{n}^{2}\left(x^{6}-1\right)}{60}\right)
\end{aligned}
$$

\subsubsection{Pressure Distribution in Tissue Region}

Solving Laplace Equation (6) using method of separation of variables and boundary and matching condition the expression for pressure distribution in tissue region is obtained as:

$$
\overline{\mathrm{P}}=\sum_{\mathrm{n}=0}^{\infty} E n \cosh \left\{\alpha_{n}(1+y)\right\} \cos \left(\alpha_{n} x\right) \cos \left(\alpha_{n} x\right)
$$

where

$$
\alpha_{n}=\frac{(2 n+1) \pi}{21}
$$

$$
\begin{aligned}
& E n=-\frac{\mathrm{P}_{0} I_{4}}{2 \alpha_{n} \cosh \left(\alpha_{n} \mathrm{H}\right)\left\{F_{4}(n)+0.5\right\}} \\
& F_{1}=\left(-\frac{3 \sigma}{\alpha^{3}}-\frac{6}{4 \alpha^{2}}+\frac{1}{4 \alpha \sigma}\right) \\
& F_{2}=\left(-\frac{3 \sigma \varepsilon}{\alpha^{4}}-\frac{6 \varepsilon}{4 \alpha^{3}}+\frac{\varepsilon}{4 \alpha^{2} \sigma}\right) \\
& F_{3}=\left(-\frac{\sigma}{\alpha^{3}}-\frac{3}{4 \alpha^{2}}+\frac{1}{4 \alpha \sigma}\right) \\
& F_{4}(n)=\left[\frac { 3 K } { H ^ { 2 } \sigma } \left\{\left(F_{1}+F_{3}\right) \frac{\mathrm{I}_{1}}{\alpha_{n}^{2}}+F_{2}\left(\mathrm{I}_{2}-\mathrm{I}_{3}\right)\right.\right. \\
& \left.\left.+\left(F_{1}+F_{3}\right) \frac{\mathrm{I}_{4} \cos \left(\alpha_{n}\right)}{\alpha_{n}^{2}}-F_{2}\left(\frac{1}{12}-\frac{\alpha_{n}^{2}}{60}\right) I_{4}\right\}\right] \\
& I_{1}=\frac{1}{2}\left[\frac{\sin 2 n \pi}{2 n \pi}-1\right] \\
& I_{2}=\left[\frac{1}{12}\left(-\frac{(-1)^{\mathrm{n}}}{n \pi}\right)+\frac{(-1)^{\mathrm{n}}}{(n \pi)^{3}}-2\left(\frac{1-(-1)^{n}}{(n \pi)^{5}}\right)\right] \\
& \cdot\left(-\frac{\sigma}{\alpha^{3}}-\frac{3}{4 \alpha^{2}}+\frac{1}{4 \alpha \sigma}\right) \\
& I_{3}=\left[\alpha_{n}^{2}\left\{\frac{1}{60}\left(-\frac{(-1)^{\mathrm{n}}}{n \pi}\right)+\frac{1}{2} \frac{(-1)^{\mathrm{n}}}{(n \pi)^{3}}\right\}\right. \\
& \left.-\frac{6(-1)^{n}}{(n \pi)^{5}}-\frac{12(-1)^{n}}{(n \pi)^{7}}+\frac{12}{(n \pi)^{7}}\right] \\
& I_{4}=\left[\frac{1-(-1)^{n}}{n \pi}\right] \text {. }
\end{aligned}
$$

\subsubsection{Concentration Distribution}

Solving Equation (13) we have obtained the expression for concentration distribution of the nutrients in tissue region as given below.

$$
c=\eta-\frac{M}{\bar{v}_{n}^{2} \mathrm{Pe}} \mathrm{e}^{-\bar{V}_{n} \mathrm{P} e y}+\frac{M}{\bar{v}_{n}} y
$$

where

$$
\bar{v}_{n}=-\frac{K R e}{H^{2}} \sum_{n=0}^{\infty} E_{n} \sinh ^{2}\left(\alpha_{n}\right) \sin \left\{\frac{(2 n+1) \pi}{2}\right\} .
$$

2.7.5. Diffusive Flux on the Capillary Tissue Interface Diffusive Flux on the wall is given as

$$
D_{F}=-D \frac{\mathrm{d} c}{\mathrm{~d} y}
$$


and expression is given as below

$$
D_{F}=-D\left[\frac{M}{\bar{v}_{n}^{2} \mathrm{P} e} e^{-\bar{V}_{n} \mathrm{P} e(y+1)}+\frac{M}{\bar{v}_{n}}\right] \text {. }
$$

\section{Results and Discussions}

Effect of deformation of the cell and permeability of the tissue on normal component of velocity, deffusive flux on the interface and concentration distribution in the tissue has been shown through the graphs. The following values of parameter are used in the model (Table 1).

Figure 2(a) represents the variation of normal component of velocity with axial distance for different values of deformation parameter $\varepsilon$. Normal component of velocity decreases with axial distance increases. As deformation parameter increases the normal component of velocity also increases. These deformations may be due to viscous stress or velocity of the fluid or due to the increased pressure developed in the gap.

Figure 2(b) shows the variation of diffusive flux with penetration depth for different value of permeability. Diffusive flux on the wall decreases as the permeability of the tissue decreases. As deformation increases, the normal component of velocity increases. Therefore diffusive flux also increases. The increase in diffusive flux is beneficial for the health of the tissue due to fact that due to increased diffusive flux nutrition supply to the deeper region also increase, therefore they get proper nutrition.

Figure 3(a) represents the normal velocity at the wall with axial distance for different values of permeability. As permeability increases normal velocity also increases. This is due to the fact that as permeability increases more fluid enters into tissue region and cells of the tissue get more nutrition.

Figure 3(b) represents the variation of diffusive flux in penetration depth for different values of deformation parameter. As deformation parameter decreases, the flux

Table 1. List of parameter used in the model.

\begin{tabular}{ccc}
\hline Parameter & Description & Value \\
\hline$K$ & Prmeability & $10^{-12} \mathrm{~m}^{2}$ \\
$\varepsilon$ & Deformation Parameter & $0.005,0.01,0.015$ \\
$\gamma$ & Radial compliance of tube & $0.001 \mu \mathrm{m} / \mathrm{mb}$ \\
$\beta$ & Radial compliance of the cell & $0.06 \mu \mathrm{m} / \mathrm{mb}$ \\
$\ell$ & Length of the capillary & $30 \mu \mathrm{m}$ \\
$\sigma$ & Slip Parameter & 0.05 \\
$\rho$ & Density of blood & $1.05 \mathrm{gm} / \mathrm{cm}^{3}$ \\
$\mu$ & Viscosity of plasma & $0.01 \mathrm{dyne} \mathrm{sec} / \mathrm{cm}^{2}$ \\
$R e$ & Reynold Number & 0.25 \\
\hline
\end{tabular}

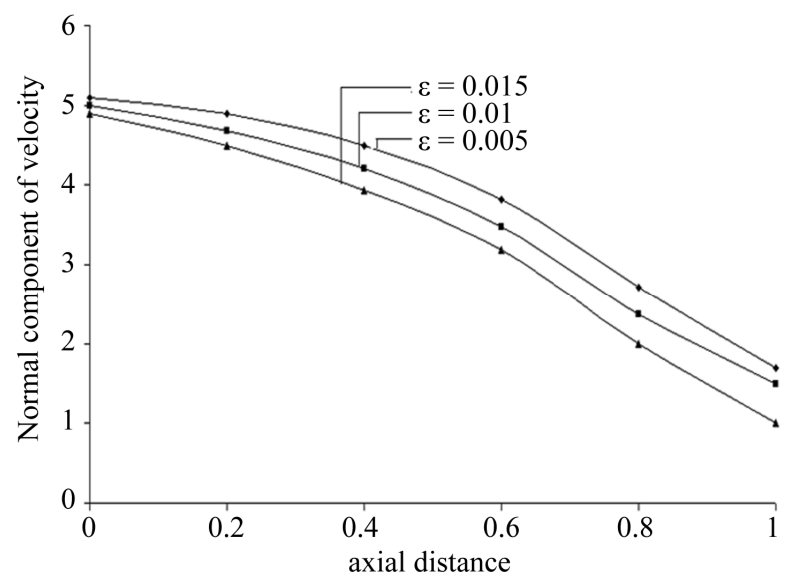

(a)

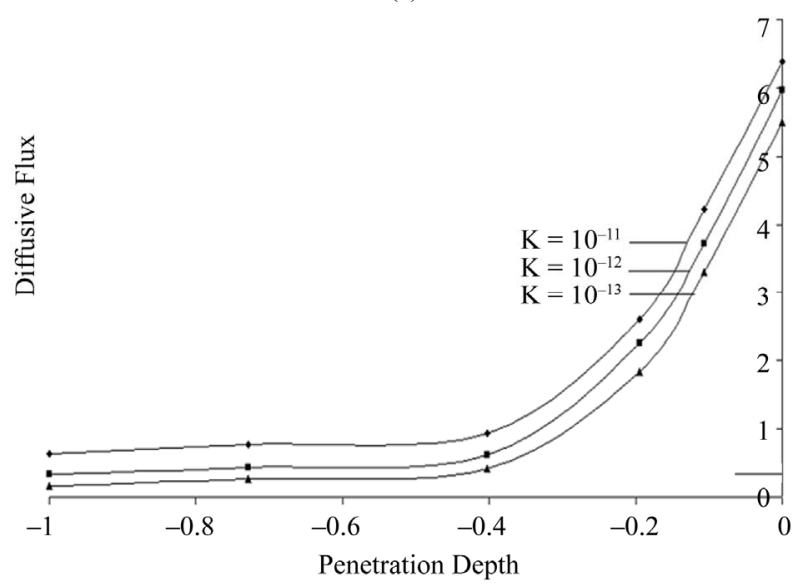

(b)

Figure 2. (a) Variation of Normal component of velocity with axial distance for different values of deformation parameter; (b) Variation of Diffusive flux with penetration depth for different values of permeability.

of dissolved material is hindered so the diffusive flux with in the tissue decreases. It also decreases in penetration depth.

Figures 4(a) and (b) describe the concentration distribution in tissue region with penetration depth for different values of permeability and deformation parameters respectively. Concentration decreases towards the no flux boundary of the tissue. When the permeability of the tissue increases the concentration increases. In this case, the cells of the tissue of the deeper region also get nutrition. Due to increase in permeability of the tissue more fluid enters into tissue so that more dissolved nutrients enter into the tissue along with the fluid. As deformation parameter increases concentration in the tissue region also increases. This is due to the fact that due to deformation, normal velocity and diffusive flux increase therefore more solute diffuses into the tissue region along with the fluid. The results are similar to those obtained by Tandon et al. $[17,18]$. 


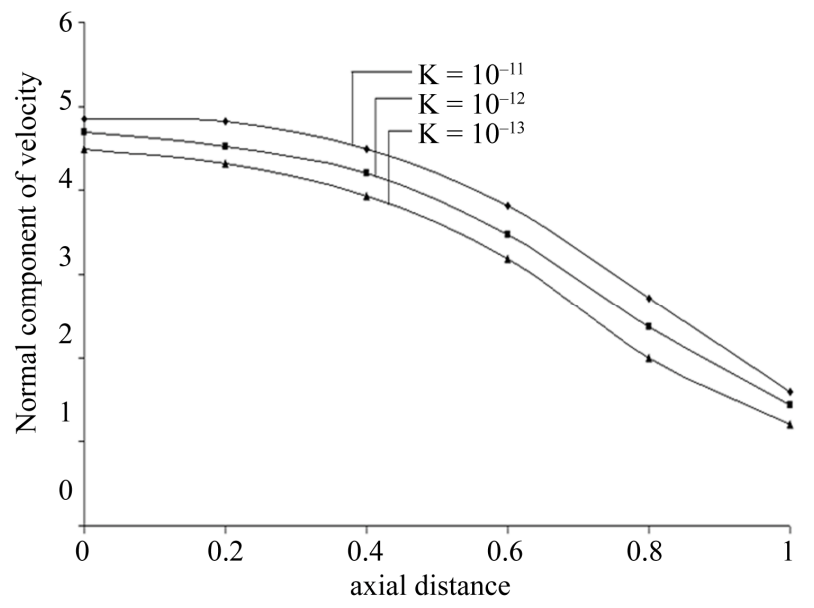

(a)

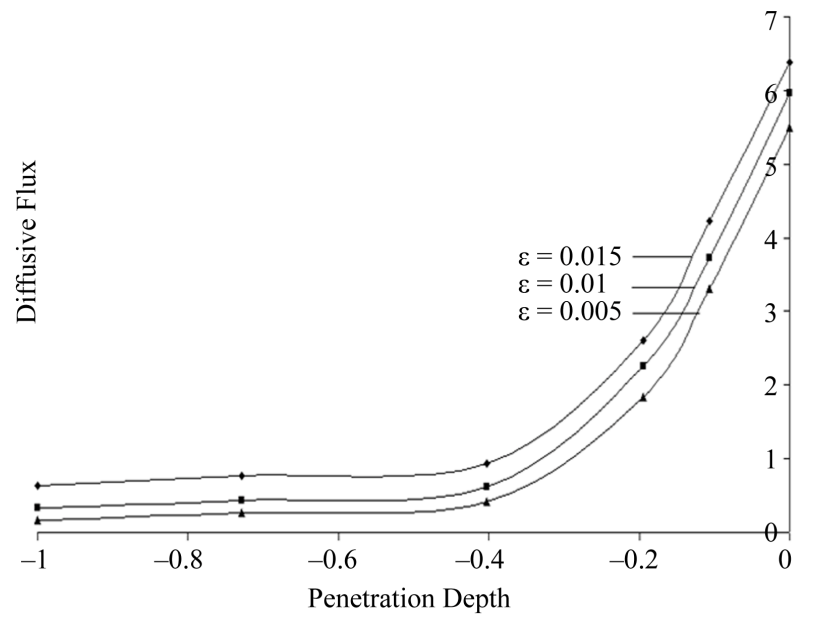

(b)

Figure 3. (a) Variation of Normal component of velocity with axial distance for different values of permeability; (b) Variation of diffusive flux with penetration depth for different values deformation parameter.

\section{Conclusions}

In this paper, we have developed a model for nutritional transport to tissue through microvessels. Our results for the deformation of the cell are useful for continuous flow of blood in capillary, so that the tissue gets proper nutrients. The studies introduce the geometry of the deformed cell in the vicinity of the porous tube wall is most important in determining the pressure drop whereas as the shape of the rest of the cell and particle spacing had minor influence on the results.

\section{Acknowledgements}

The authors are greatly indebted to Prof P. N. Tandon (Retd. Prof. and Head, Department of Mathematics, of H.B.T.I. Kanpur) for their invaluable suggestions and

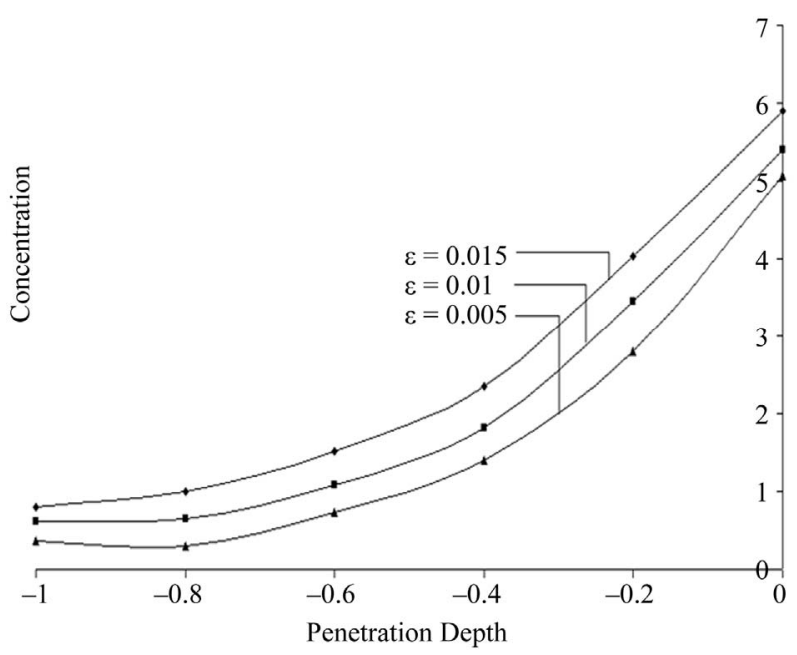

(a)

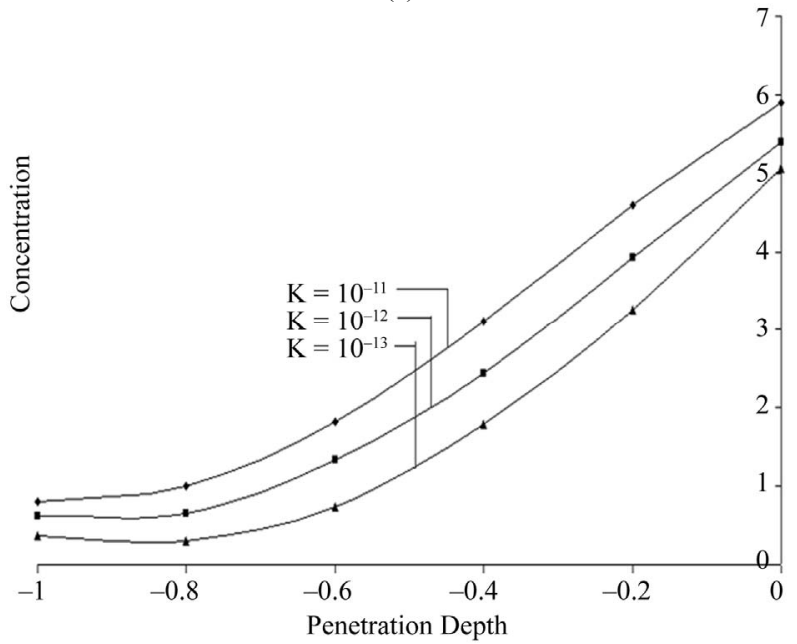

(b)

Figure 4. (a) Variation of concentration with penetration depth for different values of deformation parameter; (b) Variation of concentration with penetration depth for different values of permeability.

comments. UGC major research project $\mathrm{F}$ No. 36-320/2008 (SR) is greatfully acknowledged.

\section{References}

[1] E. M. Renkin, "Transport of Potassium - 42 from Blood to Tissue in Isolated Mammalian Skeletal Muscles," American Journal of Physiology, Vol. 197, 1959, p. 1205.

[2] N. Casson, "A Flow Equation for Pigment-Oil Suspensions of the Printing Ink Type," In: C. C. Mill, Ed., Rheology of Disperse Systems, Pergamon Press, Oxford, 1959, pp 84-104.

[3] S. E. Charm and G. S. Kurland, "Blood Rheology and Cardiovascular,” In: D. H. Bergel, Ed., Fluid Dynamics, Academic Press, London.

[4] E. C. Eringen, "Theory of Micropolar Fluids," Journal of 
Mathematical Fluid Mechanics, Vol. 16, 1966, pp. 1-18.

[5] B. B. Gupta, K. K. Nigam and H. M. Jaffrin, "A Three Layer Semi Empirical Model for Flow of Blood and Other Particulate Suspension through Narrow Tubes," Journal of Biomechanical Engineering, Vol. 104, No. 2, 1982, pp. 129-135. doi:10.1115/1.3138326

[6] H. R. Haynes, "Physical Basis of the Dependence of Blood Viscosity on Tube Radius," American Journal of Physiology, Vol. 198, No. 6, 1966, pp. 1193-2000.

[7] P. N. Tandon and R. Agarwal, "A Study of Nutritional Transport in Synovial Joints," Computers \& Mathematics with Applications, Vol. 17, No. 7, 1989, pp. 1131-1141.

[8] P. N. Tandon, M. Mishra and A. Chaurasia, "A Model for Nutritional Transport in Capillary-Tissue Exchange System," International Journal of Bio-Medical Computing, Vol. 37, No. 1, 1994, pp. 19-28. doi:10.1016/0020-7101(94)90068-X

[9] H. S. Lew and Y. C. Fung, "The Motion of the Plasma between Red Cells in the Bolus Flow," Biorheology, Vol. 6, 1969, pp. 109-119

[10] M. J. Lighthill, "Pressure Forcing of Tightly Fitting Pellets along Fluid Filled Elastic Tubes," Journal of Fluid Mechanics, Vol. 34, No. 1, 1968, pp. 113-143. doi:10.1017/S0022112068001795

[11] A. C. Barnard, L. Lopez and J. D. Hellums, "Basic Theory of Blood Flow in Capillaries," Microvascular Research, Vol. 1, No. 1, 1968, pp. 23-24. doi:10.1016/0026-2862(68)90004-6

[12] P. R. Zarda, S. Chien and R. Skalak, "Interaction of Vis- cous Incompressible Fluid with an Elastic Body," T. Belystschko and T. L. Geers, Eds., Computational Methods for Fluid-Solid Interaction Problems, American Society of Mechanical Engineers, New York, 1977, pp. 65-82.

[13] K. L. Lin, L. Lopez and J. D. Hellums, "Blood Flow in Capillaries," Microvascular Research, Vol. 5, No. 1, 1973, pp. 7-19. doi:10.1016/S0026-2862(73)80003-2

[14] T. W. Secomb and R. Skalak, "A Two Dimensional Model for the Capillary Flow of an Axisymmetric Cell," Microvascular Research, Vol. 24, No. 2, 1982, pp 194203. doi:10.1016/0026-2862(82)90056-5

[15] T. W. Secomb, R. Skalak, N. Ozakaya and J. F. Gross, "Flow of Axisymmetric Red Blood Cells in Narrow Capillaries," Journal of Fluid Mechanics, Vol. 163, 1986, pp. 405-423. doi:10.1017/S0022112086002355

[16] T. W. Secomb, R. Hsu and A. R. Pries, "Motion of Red Blood Cell in Acapillary with an Endothelial Surface Layer: Effect of Flow Velocity," American Journal of Physiology: Heart and Circulatory Physiology, Vol. 28, No. 2, 2001, pp. H629-H636.

[17] T. W. Secomb, R. Hsu and A. R. Pries, "A Model for Red Blood Cell Motion in Glycocalyx-Lined Capillaries," American Journal of Physiology: Heart and Circulatory Physiology, Vol. 274, No. 3, 1998, pp. H1016-H1022.

[18] P. N. Tandon, P. Nirmala, M. Tiwari and U. V. Rana, "Analysis of Nutritional Transport through a CapillaryNormal and Stenosed," Computers \& Mathematics with Applications, Vol. 22, No. 12, 1998, pp. 3-13. doi:10.1016/0898-1221(91)90142-Q 